\title{
FEEDBACK MODULATES THE TEMPORAL SCALE-FREE DYNAMICS OF BRAIN ELECTRICAL ACTIVITY IN A HYPOTHESIS TESTING TASK
}

\author{
M. BUIATTI, ${ }^{a, b, c 1 *}$ D. PAPO, ${ }^{\text {d,e,f1 }}$ P.-M. BAUDONNIÈRE ${ }^{g}$ \\ AND C. VAN VREESWIJK ${ }^{a}$ \\ a Laboratoire de Neurophysique et Physiologie, Université Paris Des- \\ cartes, CNRS UMR 8119, 45 rue des Saints-Pères, 75270 Paris \\ Cedex 06, France \\ ${ }^{b}$ Cognitive Neuroimaging Unit, INSERM U562, Service Hospitalier \\ Frederic Joliot, CEAIDRMIDSV, Orsay, France \\ ${ }^{c}$ Functional Neurolmaging Laboratory, Center for Mind/Brain Sci- \\ ences, Trento University, Via delle Regole 101, 38060 Mattarello, Italy \\ 'Laboratoire de Psychologie Cognitive, CNRS UMR 6146, Université \\ de Provence, Marseille, France \\ ${ }^{e}$ Wohl Institute for Advanced Imaging, Sourasky Medical Center, Tel \\ Aviv, Israel \\ ${ }^{f}$ Department of Psychology, Tel Aviv University, Tel Aviv, Israel \\ ${ }^{g}$ Neurosciences Cognitives et Imagerie Cérébrale, LENA, CNRS UPR \\ 640, Centre Hospitalier Pitié-Salpêtriere, Paris, France
}

\begin{abstract}
We used the electroencephalogram (EEG) to investigate whether positive and negative performance feedbacks exert different long-lasting modulations of electrical activity in a reasoning task. Nine college students serially tested hypotheses concerning a hidden rule by judging its presence or absence in triplets of digits, and revised them on the basis of an exogenous performance feedback. The scaling properties of the transition period between feedback and triplet presentation were investigated with detrended fluctuation analysis (DFA). DFA showed temporal scale-free dynamics of EEG activity in both feedback conditions for time scales larger than $150 \mathrm{~ms}$. Furthermore, DFA revealed that negative feedback elicits significantly higher scaling exponents than positive feedback. This effect covers a wide network comprising parietooccipital and left frontal regions. We thus showed that specific task demands can modify the temporal scale-free dynamics of the ongoing brain activity. Putative neural correlates of these long-lasting feedback-specific modulations are proposed. (c) 2007 IBRO. Published by Elsevier Ltd. All rights reserved.
\end{abstract}

Key words: scaling, EEG, hypothesis testing, ongoing brain activity, detrended fluctuation analysis.

When confronted with problems that they must solve under incomplete information, people typically generate and select hypotheses, and modify them according to feedback from the environment. Positive feedback should help in

\footnotetext{
${ }^{1}$ M.B., D.P. made equally important contributions to this article. *Correspondence to: M. Buiatti, Functional Neurolmaging Laboratory, Center for Mind/Brain Sciences, Trento University, Via delle Regole 101, 38060 Mattarello, Italy. Tel: +39-0461-883077; fax: +39-0461883066.

E-mail address: marco.buiatti@unitn.it (M. Buiatti).

Abbreviations: CRA, cluster randomization analysis; DFA, detrended fluctuation analysis; EEG, electroencephalogram; EM, eye movement; ERP, event-related potential; HT, hypothesis testing; PSA, power spectrum analysis.
}

keeping the current hypothesis active and in keeping competing hypotheses in the foreground.

Negative feedback is instrumental in shutting down neural activity associated to invalidated hypotheses, inhibiting hypothesis-feedback associations, and in promoting new hypothesis testing (HT) (Papo et al., 2003).

The modalities through which performance feedback modulates subsequent activity are still poorly understood. Studies of the time course of brain electrical activity timelocked to performance feedback using event-related potentials (ERPs) showed that positive and negative feedback conditions were associated with topographically and chronometrically separable patterns of electrical brain activity (Papo et al., 2003; Miltner et al., 1997; Ruchsow et al., 2002; Muller et al., 2005). Moreover, neuroimaging studies of performance feedback (Elliott et al., 1997; Monchi et al., 2001) showed that positive and negative feedback specifically activates separable fronto-limbico-striatal loops. Altogether, the regulatory properties of feedback were proposed to be fulfilled by a reward-related dopaminergic corticostriatal circuit (Holroyd and Coles, 2002) and its stress-driven resetting by medial temporal brain structures (Papo et al., 2003).

Both ERP and neuroimaging studies concentrated on the short-run $(<1 \mathrm{~s})$ time-locked impulse-like effects of feedback. The underlying assumption was that this time window represents the characteristic temporal scale of the response to feedback. However, contrary to perceptual phenomena, the length and content of reasoning episodes are not strictly determined by the discrete events or stimuli promoting them. In fact, reasoning comes in episodes of uneven length. This variability is inherent to the phenomenon. On one hand, imposing a fixed trial length mainly modifies decision-making thresholds (Reddi and Carpenter, 2000); on the other hand, smoothing response times through learning sessions prior to test phases essentially modifies the cognitive task actually carried out. A related issue is represented by the fact that each reasoning episode comprises a number of simultaneously active processes such as attention, working memory, and rule generation. Although each of these processes unfolds at different characteristic temporal scales, they typically interact with each other, and this renders their separation both theoretically and practically impervious.

In the present study, we investigated whether positive and negative performance feedbacks exert differential enduring modulations of electric brain activity in a $\mathrm{HT}$ task.

We addressed this issue in the following way: 1) we did not disentangle the many processes simultaneously active in $\mathrm{HT}$, but considered instead that the different cognitive 
ingredients are not separable (the whole is not just a pure addition of the parts); 2) we investigated how feedback modulates ongoing brain activity in a range of temporal scales, from those typical of single neuron activity to those allowed by the experiment.

Several studies have shown that ongoing brain activity at rest exhibits scale-free behavior: its temporal correlations are not dominated by a characteristic time scale, but rather extend with similar characteristics at all time scales up to tenths of seconds. Scale-free behavior is characterized by a power-law dependence of some measure of the system (e.g. the autocorrelation function, or the average of its fluctuations) on the scale of the system. In the case of the brain electrical activity, this means that the power-law dependence on time of one such measure is the same independently from the temporal resolution at which the observation is made, e.g. from the scale of milliseconds to the scale of seconds. This self-similar behavior is common to a large number of complex biological and non-biological systems (Stanley et al., 2000), and reflects a tendency of these systems to self-organize at different scales independently from the mechanisms underlying their dynamics (Bak et al., 1987). Scale-free behavior was shown for electroencephalogram (EEG) signal amplitudes (Novikov et al., 1997; Pereda et al., 1998; Watters, 1998; Freeman and Barrie, 2000; Hwa and Ferree, 2002; Le Van Quyen, 2003), for amplitudes of alpha and beta band oscillations (Linkenkaer-Hansen et al., 2001, 2004; Nikulin and Brismar, 2005), and for the duration of synchrony episodes between different brain areas in several frequency bands (Gong et al., 2003; Stam and de Bruin, 2004). Spatial scale-free dynamics was also shown at scales ranging from a few millimeters to an entire hemisphere (Freeman and Barrie, 2000). A few recent studies showed that scalefree dynamics is not only an intrinsic physiological property of spontaneous ongoing brain activity, but is modulated (and not disrupted) by passive sensory nerve stimulations (Linkenkaer-Hansen et al., 2004) or eye open/eye closed condition (Stam and de Bruin, 2004). Two more studies examined the scale-free dynamics of the ongoing activity in several frequency bands relative to different cognitive tasks (music listening compared with text listening, a spatial imagination task and a rest condition (Bhattacharya and Petsche, 2001), imaginary and real visual-motor tracking (Popivanov et al., 2006)) and found again that temporal scaling is modulated, but not disrupted, by different tasks.

Following these latter studies, we conjectured that performance feedback may modulate the scale-free dynamics of the ongoing brain activity associated with the subsequent performance of a complex cognitive task. To investigate this hypothesis, we re-analyzed the data from the EEG study of HT performed by Papo et al. (2003). In this task, subjects serially tested hypotheses concerning a hidden rule by judging its presence or absence in triplets of digits, and revised them on the basis of an exogenous performance feedback. We focused on the period during which subjects, after having received either a positive or a negative feedback, are adjusting their hypotheses in order to optimally respond to the following triplet. We hypothe- sized that positive and negative feedbacks induce two separable dynamic regimes characterized by a quantitatively different scale-free behavior of the underlying ongoing brain activity during preparation of the response to a new triplet. In order to avoid the influence of the highamplitude transitory ERP evoked by feedback on the estimation of the scale-free behavior, we analyzed the temporal segment starting from $1.5 \mathrm{~s}$ after feedback, i.e. well after the extinction of the ERPs evoked by the feedback, and ending just before the new triplet presentation. Evoked responses have been shown to modestly affect long-range correlations in ongoing activity, provided long enough time series are taken into account (Linkenkaer-Hansen et al., 2004). However, since the time interval considered in our study was of very limited length, our analysis tried to stay as far away as possible from the perceptually-evoked response, lest the whole interval of interest be dominated by the high amplitude of the early evoked potentials.

We investigated both the existence of scale-free dynamics of the raw EEG activity and its modulation by feedback with detrended fluctuation analysis (DFA) (Peng et al., 1995), a method that estimates how the average temporal fluctuations of the signal at a particular time scale depend on that scale: a power-law dependence is the sign of scale-free behavior. DFA is a widely used estimator of scale-free behavior in biological time series because it is robust to spurious correlation detection induced by nonstationarities in the signal (Peng et al., 1995).

\section{EXPERIMENTAL PROCEDURES}

\section{Subjects}

Thirteen right-handed graduate and undergraduate students volunteered in the experiment. Subjects had normal or corrected to normal vision and no history of neurological or psychiatric disease. Three of them could not be kept for further analyses due to excessive rates of recording artifacts; while one subject was excluded because she was not naive as to the task's manipulations. The nine remaining subjects (five women and four men; mean age $=25$, range $21-30$ years) were all blind as to the experiment's objectives.

\section{Stimuli and procedure}

Subjects sat in front of a computer screen and judged, using a double-button press device, whether or not triplets of numbers were instances of a hidden rule chosen by the experimenter. At the beginning of each trial (Fig. 1) one triplet was presented. Subjects' responses appeared on the screen below the triplet, as an "O" and an "N" respectively for positive and negative responses. Following a time interval varying between 800 and 1200 $\mathrm{ms}$, subjects received the experimenter's feedback on the computer screen, indicating whether their performance was correct or not. Positive and negative feedbacks were respectively represented by a green and a red square surrounding the letters symbolizing subjects' response. Triplet, subjects' response, and feedback stayed on the screen till $1 \mathrm{~s}$ after feedback onset. The screen was then offset before the following trial could start. There was a $3-5 \mathrm{~s}$ intertrial interval. There were 30 blocks of 10 trials each, corresponding to 30 different hidden rules. Subjects were informed that the rule was changed at the end of each block of trials. Successive blocks were separated by time intervals of the order of $30 \mathrm{~s} / 1 \mathrm{~min}$. Importantly, the feedbacks were controlled by 


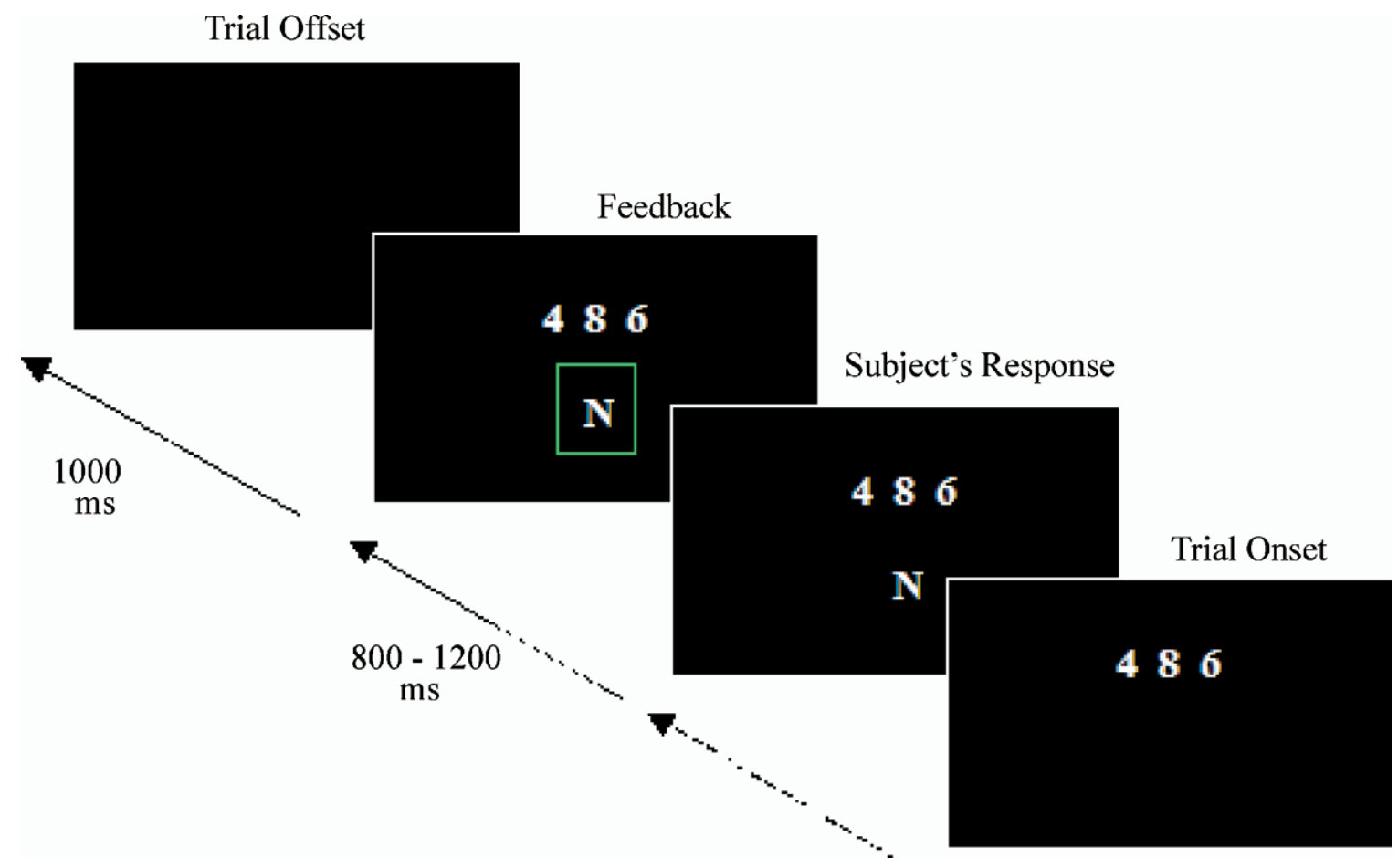

Fig. 1. Example of a trial with positive feedback. The period analyzed in this study started from $1.5 \mathrm{~s}$ after feedback ( $0.5 \mathrm{~s}$ after trial offset), i.e. well after the extinction of the ERPs evoked by feedback, and ended just before the new triplet presentation (marked as trial onset in the figure).

the experimenter and not by subject's performance, as there were no rules behind the triplets. Feedback frequency was manipulated, so that an equal number of blocks respectively had 8:2, 5:5, and $2: 8$ positive-to-negative feedback ratios. In the (8:2) and $(2: 8)$ blocks the last five trials were paired to five consecutive positive and negative feedbacks respectively, while in all blocks the first five trials comprised either two or three positive/negative feedback responses. The order of presentation of blocks was quasi-randomized across subjects. All subjects were verbally debriefed after the experimental session, and none of them was aware of feedbacks being unrelated to performance. Thus, it can safely be stated that, for all practical purposes, no subject construed feedback as random, with all subjects behaving accordingly and trying to the best of their abilities to carry out the proposed hypothesis testing task. Importantly, analysis of variance revealed that response times increased as a function of the frequency of negative feedback. The fact that response times varied according to feedback frequency convincingly indicated the meaningfulness of this experimental manipulation (cf. Papo et al., 2003, Behavioral Results). Behavioral results and analysis of ERPs time-locked to feedback presentation as a function of the feedback value (positive or negative) are extensively described and discussed in Papo et al. (2003). The core result of the ERP study is that positive and negative feedback conditions were associated with topographically and chronometrically separable patterns of electrical brain activity. Positive trial-to-trial feedback elicited a parieto-central P300, whereas negative feedback was characterized by P200N240 complexes with a frontal-central onset and lasting longer than in posterior regions.

\section{EEG recording system}

Brain electrical activity was recorded from 62 electrodes positioned according to the extended 10-20 system location, with a nasion reference. The electro-oculogram (EOG) was also recorded. The EEG was amplified $(0.05-100 \mathrm{~Hz})$ with a sampling frequency of $500 \mathrm{~Hz}$. The analyses performed in this work are restricted to the innermost 39 electrodes shown in Fig. 2.

\section{Pre-processing and data epoching}

Main analysis. Raw EEG data were corrected for blink, vertical and horizontal eye movements (Gratton et al., 1983). Epochs of $2.5 \mathrm{~s}$ beginning $1.5 \mathrm{~s}$ after feedback presentation and

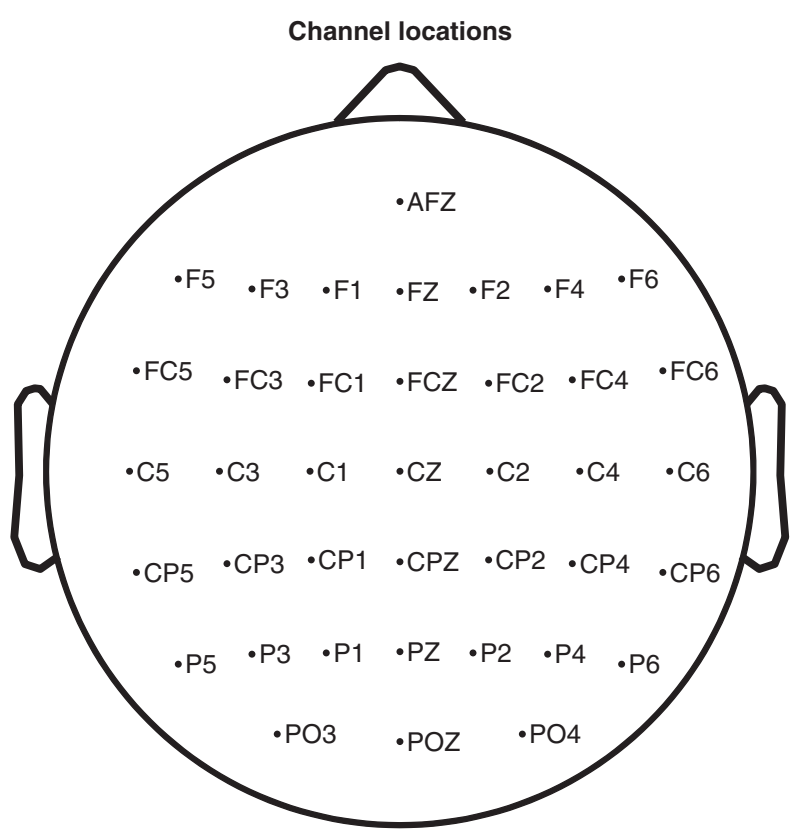

Fig. 2. Channel location of the 39 electrodes used in this study. 
ending $4 \mathrm{~s}$ after feedback presentation were extracted from the corrected data. Since triplet presentation occurred 4-6 s after feedback presentation, the selected temporal segment is the largest segment of a fixed length ending before the arrival of the next triplet for all the trials. Since for each block, EEG recordings stop at feedback offset of the last trial, epochs were extracted from the first nine trials of every block, while no data were available for the preparation period following the last trial. Artifacted epochs were discarded by a semi-automatic artifact rejection procedure (threshold $\pm 80 \mu \mathrm{V}$ ). After pre-processing, on average 100 trials for each electrode, subject and condition were available for further analyses.

Control analysis. Due to the duration of the analyzed epochs $(2.5 \mathrm{~s})$, epochs containing at least one eye movement were relatively frequent. Although residuals of corrected eye movements are unlikely to affect the results of the analyses carried out in this study, in order to rule out any influence of eye movements and/or the eye movement correction algorithm on the results, a control analysis was directly performed on eye-movement-free raw data. Epochs of $2 \mathrm{~s}$ beginning $2 \mathrm{~s}$ after feedback presentation and ending $4 \mathrm{~s}$ after feedback presentation were extracted from the raw EEG data. As for the main analysis, epochs were extracted from the first nine trials of every block. Epochs containing both eye movements and other artifacts detected by a semiautomatic artifact rejection procedure (threshold $\pm 80 \mu \mathrm{V}$ ) were discarded. As a result, two more subjects were discarded because of the high rate of epoch rejection. An average of 30 artifact-free trials for each electrode, subject and condition was available for further analyses.

\section{Estimation of scale-free behavior}

The most intuitive way to characterize scale-free behavior of a time series $x(t)$ is by looking at its temporal correlations, quantified by its autocorrelation function:

$$
C_{x}(\tau)=\frac{\langle(x(t)-\langle x\rangle)(x(t-\tau)-\langle x\rangle)\rangle}{\left\langle(x(t)-\langle x\rangle)^{2}\right\rangle} .
$$

The autocorrelation function (1) is a measure of the "memory" of the time series, indicating how much the signal $x(t)$ depends on its past value $\tau$ time steps before. This memory can be grossly quantified with the correlation time $T=\int_{0}^{\infty} C_{x}(t) d t$, representing the characteristic time scale of the system. If $T$ is finite, the system loses its memory for times $t \gg T$. If however the autocorrelation function decays as

$$
C_{x}(t) \propto t^{-\beta} \quad \text { for } t \rightarrow \infty
$$

with $0<\beta<1$, then $T$ diverges and no characteristic time scale exists. In this case, the time series $x(t)$ possesses long-range scale-free correlations characterized by the scaling exponent $\beta$. Estimating $\beta$ from the power-law tail of the autocorrelation function is not the best method though, since noise and slow trends often distort the result. Moreover, temporal correlations in EEG time series are typically stronger than those described by Eq. 2. In this regime, called non-ergodic, the autocorrelation function cannot be defined anymore because it does not converge to the ensemble average for each temporal lag, but randomly and widely fluctuates around the average (Margolin and Barkai, 2005). However, some other measures as those estimated by DFA and power spectrum analysis (PSA) may still exhibit power-law scale-free behavior.

\section{DFA}

DFA was introduced to characterize the scale-free behavior of non-stationary time-series (Peng et al., 1994, 1995). We adapted DFA to our data statistics by the following three consecutive steps:
1) The time series $x(t)$ of length $N$ is integrated:

$$
y(t)=\sum_{t^{\prime}=1}^{t}\left(x\left(t^{\prime}\right)-\langle x\rangle\right)
$$

2) The integrated time series is divided into segments overlapping for one half of their length $n$ (we verified that partial overlapping, used to having better statistics for long segments, does not alter the estimate; Herzel et al., 1994). For each segment, a least-squares line is fit to the data, representing the trend in that segment. The y coordinate of the straight line segments is denoted by $y_{n}(t)$. Single-segment residuals are then obtained by subtracting the trend $y_{n}(t)$ from the integrated time series.

3) The root mean square fluctuation of the residuals is computed by

$$
F(n)=\sqrt{\frac{1}{2(N / n)-1} \sum_{t_{0}}\left(\frac{1}{n} \sum_{t=t_{0}}^{t_{0}+n}\left[y(t)-y_{n}(t)\right]^{2}\right)}
$$

where $t_{0}$, denoting the beginning of each segment, increases in jumps of $n / 2$ along the sequence. Simulations (Peng et al., 1994; Buldyrev et al., 1995) and analytical studies (Taqqu et al., 1995; Heneghan and McDarby, 2000) have shown that $F(n)$ has the asymptotic behavior:

$$
F(n) \propto n^{\alpha}
$$

where $\alpha=0.5$ for processes with a finite characteristic scale, while

$$
\alpha=1-\frac{\beta}{2}
$$

if the autocorrelation function decays like in Eq. (2) with $0<\beta<1$. One major advantage of DFA over other methods is that if the system exhibits scale-free dynamics in the non-ergodic regime, DFA residuals still keep the power-law behavior of Eq. (4): $\alpha=1$ for $1 / f$ noise, and $\alpha=1.5$ for brownian noise. Since EEG time series exhibit power-law temporal scaling with $\alpha>1$ (Watters, 1998; Lee et al., 2002), this property was crucial to our analysis.

For the main analysis, the segment length $\mathrm{n}$ ranged from $8 \mathrm{~ms}$ to $2.5 \mathrm{~s}$, while for the control analysis it ranged from $8 \mathrm{~ms}$ to $2 \mathrm{~s}$. In order to have a sufficient statistics, for every subject the single-trial mean square residuals were further averaged across all blocks over all trials corresponding to the same feedback type. The critical exponent $\alpha$ was estimated by computing the slope of a linear least-square fit of $F(n)$ on a log-log scale in the scaling range. As shown in the Results, the scaling range was restricted to $156-1248 \mathrm{~ms}$ for the main analysis, and to 156-998 ms for the control analysis.

\section{PSA}

In order to compare DFA to an alternative method for the detection of scaling, PSA was also used. The theoretic power spectrum of a process $x(t)$ is defined as

$$
S(f)=\left|\int_{-\infty}^{\infty} x(t) e^{-2 \pi i t} d t\right|^{2}
$$

The power spectrum is linked to the autocorrelation function by a Fourier transform. If the correlation time $T$ of the process $x(t)$ is finite, the power spectrum $S(f)$ tends to a finite value for $f \rightarrow 0$. If however the autocorrelation function decays like in Eq. (2) with $0<\beta<1$, the power spectrum diverges for $f \rightarrow 0$ as

$$
S(f) \propto f^{-\gamma}
$$


where

$$
\gamma=1-\beta
$$

(Rangarajan and Ding, 2000). The relation between $\gamma$ and the exponent $\alpha$ estimated by DFA is obtained by comparing Eq. (5) and Eq. (8):

$$
\alpha=\frac{1+\gamma}{2}
$$

As DFA residuals, if the system's dynamics is scale-free, the power spectrum still shows a power-law behavior in the nonergodic regime, corresponding to the exponent $\gamma$ growing larger than 1. In fact, Heneghan and McDarby (2000) showed that PSA and DFA are theoretically equivalent measures. Therefore, PSA represents a valid alternative method to DFA to estimate the temporal scaling of EEG time series (Pereda et al., 1998; Freeman and Barrie, 2000; Le Van Quyen, 2003).

The power spectrum of the EEG time series was estimated by evaluating the power spectrum on every trial, and averaging across all trials. Single-trial power spectrum was estimated by its periodogram: each trial was divided in windows overlapping for one half of their length, the power spectrum of every window was computed as the square modulus of the fast Fourier transform (FFT) estimate, and the resulting values were averaged across all windows. The length of the window was half the length of the trial. We checked that no substantial difference emerged when using smoothing windows. As for DFA, for every subject single-trial power spectrum estimates were averaged across all blocks over all trials corresponding to the same feedback type. The critical exponent $\gamma$ of Eq. (7) was then estimated by computing the slope of a linear least-square fit of the periodogram on a log-log scale in the scaling range. The frequency domain equivalent of the DFA scaling range was $0.8-6.4 \mathrm{~Hz}$. The corresponding exponent $\beta$ could then be computed through Eq. (8).

\section{Statistical analysis}

Statistical analysis of the difference between the scaling exponents in the two experimental conditions was performed by cluster randomization analysis (CRA) Fieldtrip software for EEG/MEG analysis, freely available at http://www.ru.nl/fcdonders/fieldtrip, a statistical method that overrides the multiple comparison problem in comparing topographies of EEG measures (Takashima et al., 2006). In its original formulation, CRA was conceived to analyze statistical differences in the ERPs/fields or in the time-frequency representation between two experimental conditions in EEG/MEG experiments. In our study, CRA on scaling exponents consisted of two steps: 1) A paired $t$-test was computed for every electrode between the scaling exponents of all subjects in the two conditions. The $t$-statistics array was then thresholded at $P=0.05$ and passed as an argument to a cluster-finding algorithm to find all clusters of connected electrodes exceeding the threshold. The cluster-finding algorithm eliminates small isolated (hence physiologically not plausible) clusters by setting a minimum number of above-threshold neighboring electrodes to belong to a cluster (set to two in our study). 2) For every cluster, the cluster-level test statistics was computed as the sum of the $t$-statistics of every electrode belonging to that cluster. The null distribution (namely the distribution of the test statistic under the null hypothesis) of the maximum of the different cluster-level test statistics was computed by randomly permuting the order of paired observations. $P$-values for the cluster-level statistics under this distribution of the maximum were then computed. These $P$-values represented the probability that the cluster having the maximum cluster-level statistics belongs to the null distribution. Clusters having $P$-values $<0.05$ were considered statistically significant in this study.
CRA on the power spectrum was computed following the same steps described above, with the only difference that the $t$-statistics array of step 1 had an additional dimension (frequency). Clusters could therefore extend over several frequency bins.

\section{Surrogate data}

In order to prove DFA and PSA efficiency in estimating the scaling exponents, surrogate time series were generated having scalefree dynamics with scaling exponents in the same range of those estimated in previous studies on raw EEG brain activity (Watters, 1998; Pereda et al., 1998; Lee et al., 2002; Le Van Quyen, 2003). For the sake of simplicity, we generated dichotomic time series having either the value 1 or -1 at each time step. We followed the procedure used in Buiatti et al. (1999). The sequence $y_{i}$ of random numbers uniformly distributed in the interval $[0,1]$ was first transformed into the sequence $\tau_{i}$ defined by

$$
\tau_{i}=T\left(\frac{1}{y_{i}{ }^{(\mu-1)}}-1\right)
$$

with $\mu>1$. The sequence $\tau_{i}$ is characterized by the distribution density $\psi(\tau)$ given by

$$
\psi(\tau)=(\mu-1) \frac{T^{\mu-1}}{(\tau+T)^{\mu}}
$$

The surrogate time series were generated from the sequence $\tau_{i}$ by the following steps: for every $i$, a subsequence of $n=\left[\tau_{i}\right]+1$ (where [...] indicates the integer part) identical $1 \mathrm{~s}$ are added to the time series $x(t)$, and a sign (1 or -1$)$ is randomly assigned to the subsequence. In the simulations, the value $T=0.5$ was used as in (Buiatti et al., 1999). The resulting time series consists of a sequence of laminar regions (namely, subsequences having an identical value at all times) whose length has the power-law distribution of Eq. (11). In the range $2<\mu<3$, the autocorrelation function of such time series has a power-law decay as in Eq. (2), where the relation between the two exponents is $\beta=\mu-2$ (Buiatti et al., 1999). In order to check the efficiency of DFA and PSA in estimating the temporal scaling in the non-ergodic regime $1<\mu<2$, we extended the relations between the three exponents, $\alpha=(4-\mu) / 2$ and $\gamma=3-\mu$ to the non-ergodic regime $1<\mu<2$. The physical meaning of this extension was investigated in Kalashyan et al. (in press).

\section{RESULTS}

\section{Testing DFA and PSA on surrogate data}

We first tested on surrogate data the efficiency of DFA and PSA to estimate the correct scaling with our experimental data statistics. As will be shown in the next section, the scaling range over which scaling exponents are estimated is restricted to the interval 156-1248 ms. For every value of the scaling exponent $\alpha$ between 0.7 and 1.3, we generated 100 independent groups of 70 time series (corresponding to the minimum number of observations per subject and condition in the main analysis), temporally correlated with the scaling exponent $\alpha$ (see Experimental Procedures), each one as long as the experimental trials (1250 time steps). Scaling exponents were estimated for each one of the 100 groups of trials by following the same averaging and fitting procedure described in the Experimental Procedures for the main analysis, and by using the same time and frequency ranges to compute the fit. 
Results of the simulation are shown in Fig. 3. DFA clearly gives a very good estimate for every value of $\alpha$, while PSA tends to increasingly overestimate $\alpha$ for increasing $\alpha$. Similar results (not shown) are obtained for the statistics of the control analysis, though due to poorer statistics, standard deviations are larger. We therefore used DFA to estimate the scaling exponents of the data.

A supplementary check of DFA efficiency was performed by computing DFA on the experimental data after randomly shuffling their temporal order (this corresponds to disrupting temporal correlations while leaving the data distribution unaffected). As theoretically predicted in the case of no correlations, DFA gave an average scaling exponent $\alpha=0.50 \pm 0.01$ (average \pm standard deviation over all electrodes, subjects and conditions), and no statistically significant difference between positive and negative condition was detected (CRA gave no significant clusters).

\section{Scaling range}

We investigated whether, and in which temporal range, temporal correlations in the data exhibited power-law scaling. As shown in the example of Fig. 4, DFA residuals exhibit a different behavior at two different time ranges: they show no scaling in the short-time range up to about $\tau=150 \mathrm{~ms}$, while they are very well fitted by a power law for times $\mathrm{t}>\tau$ (the dotted line in the figure indicates the leastsquare fit to the data in the range 156-1248 ms). Scaling exponents are generally larger than 1 for most subjects and electrodes, indicating a non-ergodic dynamical regime consistent with previously reported values (Watters, 1998;
Pereda et al., 1998; Lee et al., 2002). For times larger than $1248 \mathrm{~ms}$, the trial's finite size causes the power-law fit to become highly irregular. This is most probably due to a lack of statistical power: only one or two residual estimates are computed from each trial in this range. We verified that this behavior is common to all subjects, electrodes and conditions. For every electrode, subject and condition, we therefore selected the range 156-1248 ms to compute the associated scaling exponent.

\section{Feedback modulates the scaling exponent}

DFA analysis shows that feedback indeed modulates the scale-free dynamics of the ongoing activity: as illustrated by the slopes of the DFA residuals in the example of Fig. 5, DFA scaling exponents of the EEG signals following negative feedback are higher than the ones following positive feedback.

Fig. 6 summarizes the topography of the scaling exponents and the statistical significance of feedback modulation. Scaling exponents are higher in anterior regions than in posterior ones in both conditions in a similar fashion (left-hand and central scalp of Fig. 6). The difference between the two exponents in the two conditions, though small compared with the exponent variability across electrodes, is very coherent on wide brain areas (right scalp of Fig. 6). CRA gives a highly significant two-lobes cluster $(P=0.002)$ composed by a middle posterior area and a left anterior one. No other statistically significant clusters arise from CRA.

Scaling exponents also widely vary among subjects: the average of the exponents over all electrodes for every

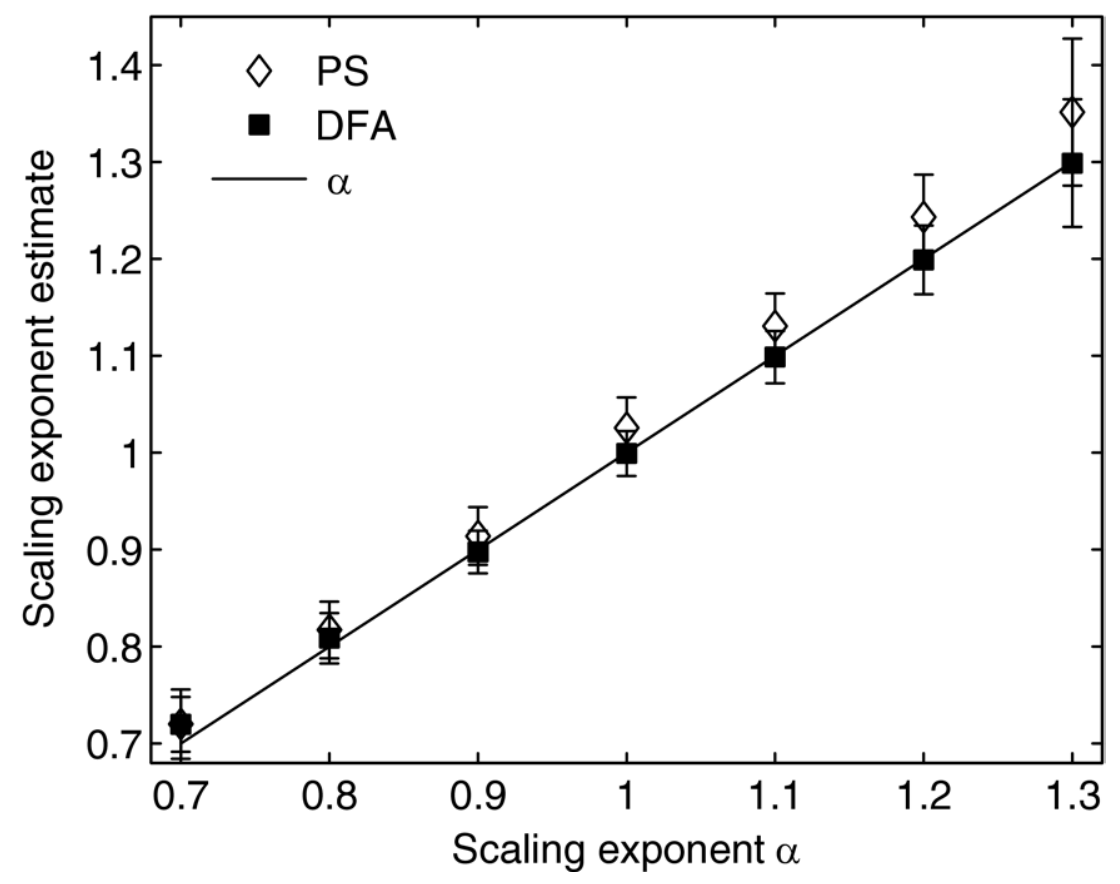

Fig. 3. Plot of the average DFA estimates and average PSA estimates over 100 sets of surrogate data of the scaling exponent $\alpha$ versus the true scaling exponent $\alpha$ used to generate the time series. Error bars represent the standard deviation of the mean. The segmented line represents $\alpha=\alpha$. DFA clearly gives a very good estimate for every value of $\alpha$, while PSA tends to increasingly overestimate $\alpha$ for increasing $\alpha$. 

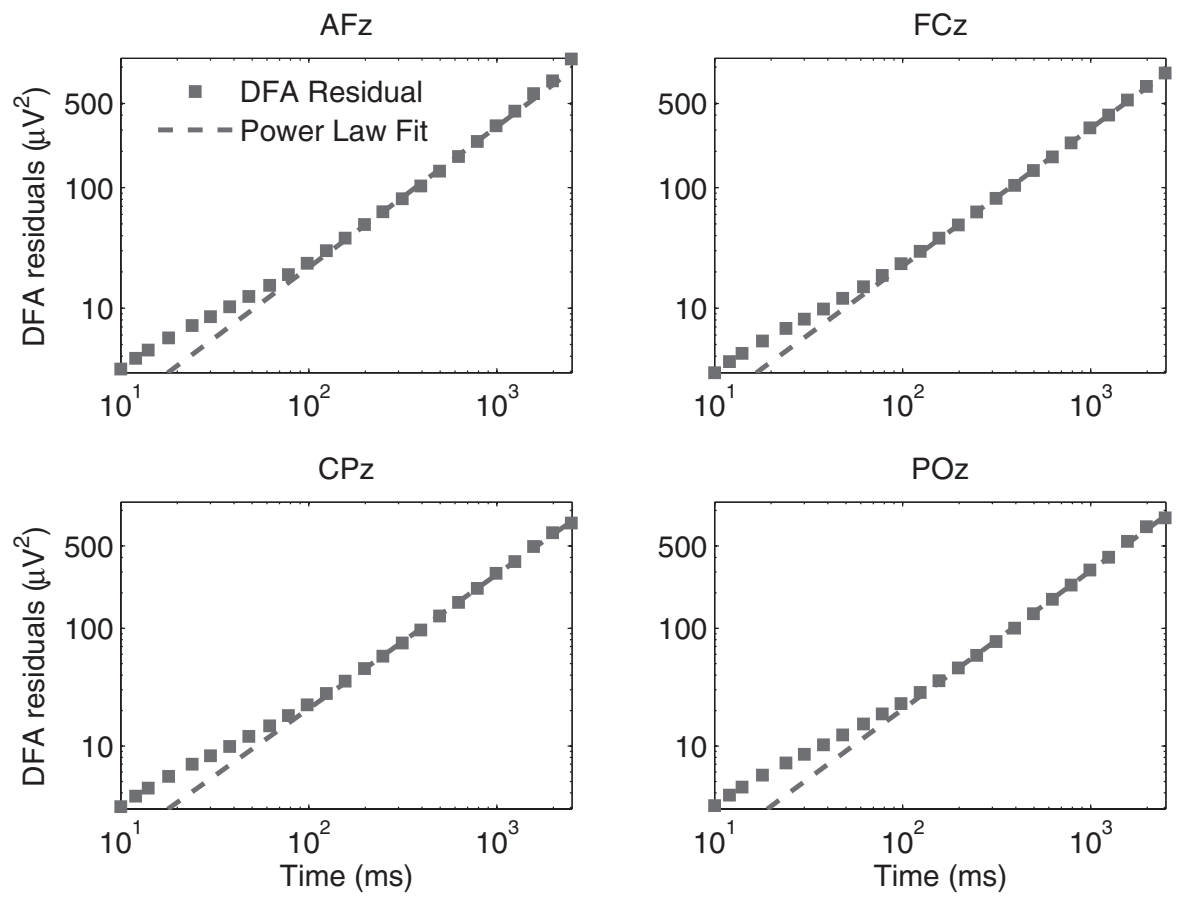

Fig. 4. DFA residuals $F(n)$ as a function of time window length $n$ (squares) in log-log scale, and linear least-square fit to $F(n)$ in the range $156-1248$ $\mathrm{ms}$ (dashed line), relative to electrodes AFZ, FCZ, CPZ and POZ, subject 5, condition of positive feedback. DFA residuals exhibit robust power law scaling in the range 156-1248 ms, while at shorter and longer time scales their behavior is irregular. Scaling exponent estimates are $\alpha=1.17, \alpha=1.14$, $\alpha=1.14, \alpha=1.18$, respectively.

subject fluctuates between $\alpha=1.03$ and $\alpha=1.39$. However, feedback modulation is very coherent within every subject: the average exponent over the significantly different elec-
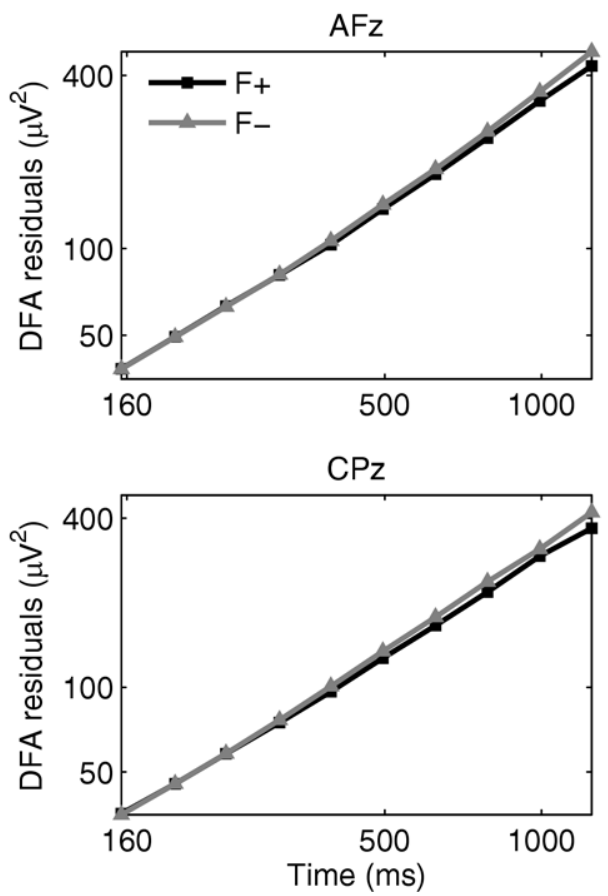

trodes is higher for negative feedback than for positive feedback in eight subjects out of nine, and substantially equal in the remaining subject (Fig. 7).
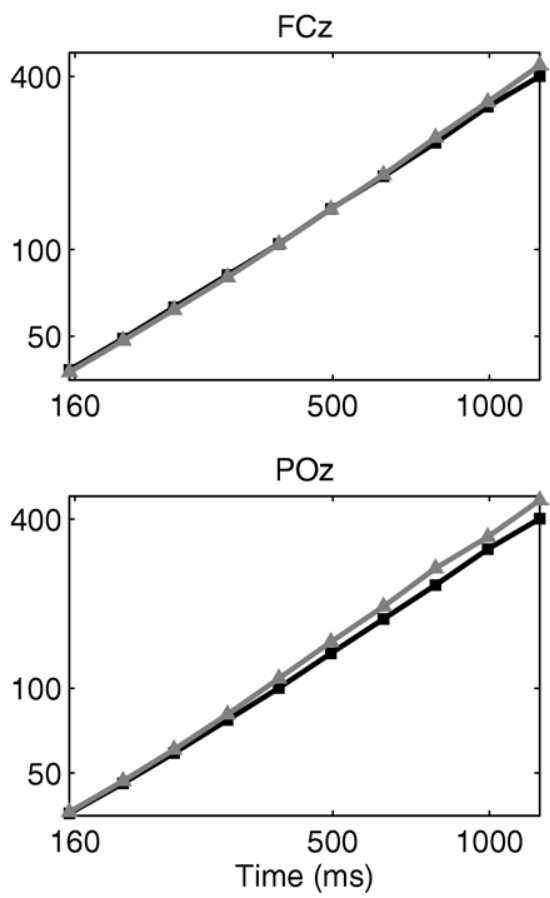

Fig. 5. Log-log plots of DFA residuals $F(n)$ as a function of time window length $n$ averaged over positive (black squares) and negative (gray triangles) feedback conditions relative to electrodes AFZ, FCZ, CPZ and POZ, subject 5 . The slopes of DFA residuals are markedly higher in the negative feedback condition than in the positive feedback one. 

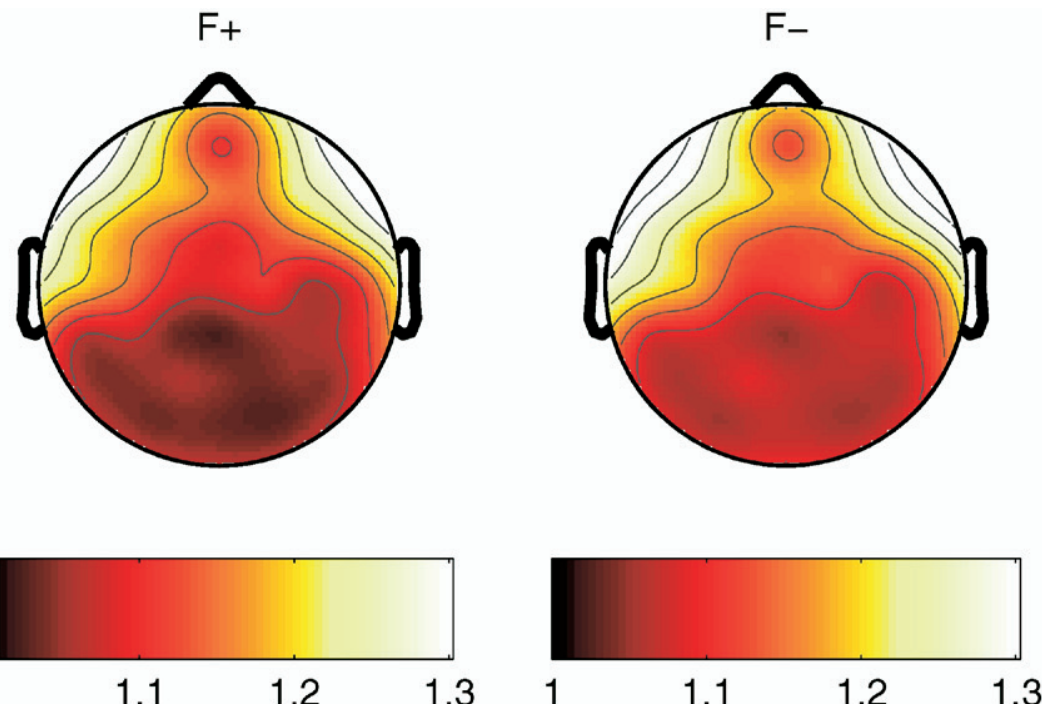
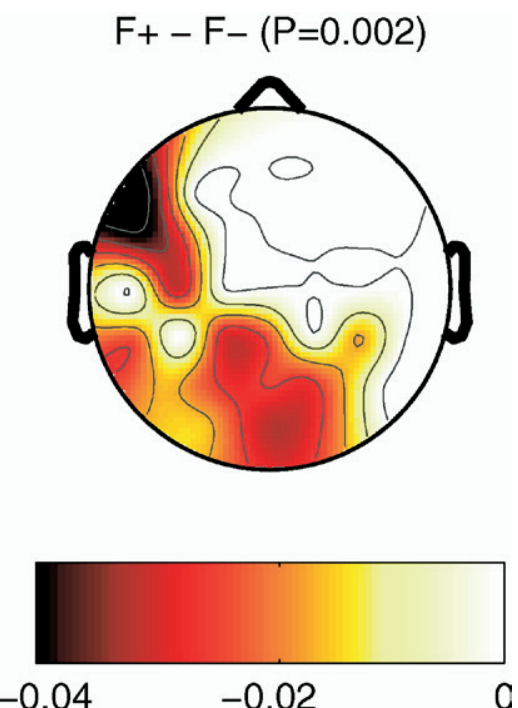

Fig. 6. Topography of DFA scaling exponents averaged across all subjects for positive (left-hand scalp) and negative (middle scalp) feedback condition. Color bars indicate the correspondence between colors and scaling exponent values. The right-hand scalp shows the topography of the statistically significant cluster resulting from CRA $(P=0.002)$, indicating a wide brain area in which negative feedback scaling exponents are significantly higher than positive feedback ones. The cluster comprises 17 electrodes out of 39 . Colors for the electrodes belonging to the cluster indicate the difference of the averaged scaling exponents between positive feedback and negative feedback (see color bar). A zero difference is assigned to the electrodes not belonging to the cluster. For interpretation of the references to color in this figure legend, the reader is referred to the Web version of this article.

\section{Feedback does not modulate power spectrum}

We investigated whether feedback also modulates the EEG power spectrum at any specific frequency band. Data power spectrum is characterized by a low-frequency powerlaw decrease followed by a prominent alpha peak around $10 \mathrm{~Hz}$ in most posterior electrodes (Fig. 8). However, no feedback modulation is visible. CRA on all frequency bands in the interval $0.05-90 \mathrm{~Hz}$ did not reveal any significant cluster in any frequency band $(P>0.12)$, meaning no statistically significant difference between the two conditions in the whole EEG power spectrum.

\section{Controlling the influence of eye movements}

Due to the duration of the analyzed epochs (2.5 s), epochs containing at least one eye movement (EM) were relatively frequent. Despite the fact that residuals of EM surviving the artifact correction algorithm are unlikely to affect the results of the analyses carried out in this study, in order to explicitly rule out any influence of eye movements and/or of the eye movement correction algorithm on the results, we investigated whether: 1) feedback modulates EM; and 2) feedback modulation of the scaling exponent is attributable to the effect of EM.

\section{Feedback modulates eye movements}

Since we were interested in the amplitude of EM, but not in its sign, we measured the average of the absolute value of vertical and horizontal EM in the two conditions over all trials. We found that EM were quite frequent within about $800 \mathrm{~ms}$ after screen offset (hence in the first $300 \mathrm{~ms}$ of the time window explored in the main analysis), but quite rare in the rest of the period before triplet presentation. How- ever, vertical EM were slightly but significantly larger for negative feedback than for positive feedback (paired $t$-test on the single subject absolute value averaged over the time window $1.5-4 \mathrm{~s}$ of the main analysis, $P<0.03$ ), while horizontal EM were not $(P>0.18)$.

\section{Feedback modulation of scaling exponents is independent of EM}

In order to rule out the influence of EM residuals after the artifact correction algorithm on the feedback modulation of the scaling exponents, we recomputed the scaling exponents on the raw data after removal of all epochs containing EM (see Control Analysis in the Experimental Procedures for details). In this EM-free data set, no statistical difference was detectable between the residual EM signals of the two feedback conditions: paired $t$-test on the single subject absolute value averaged over the time window $2-4$ $\mathrm{s}$ of the control analysis gave $P>0.4$ for both vertical and horizontal eye movements. Compared with the main analysis, data statistics were substantially reduced (seven subjects, an average of 30 trials per subject). However, simulations on surrogate data (see first section of Results) showed that the statistics were sufficient for a reliable estimate of the scaling exponents by DFA. The topography of the scaling exponents estimated from the EM-free data set (left and middle scalp in Fig. 9) showed a very similar distribution of both positive and negative feedback exponents to the one emerging from the main analysis, the only difference being a decrease of the most fronto-lateral exponents. The statistical analysis of the difference between the two conditions also confirmed the results of the first analysis (right scalp in Fig. 9): CRA revealed a two-lobes cluster consisting of a left anterior and middle posterior 


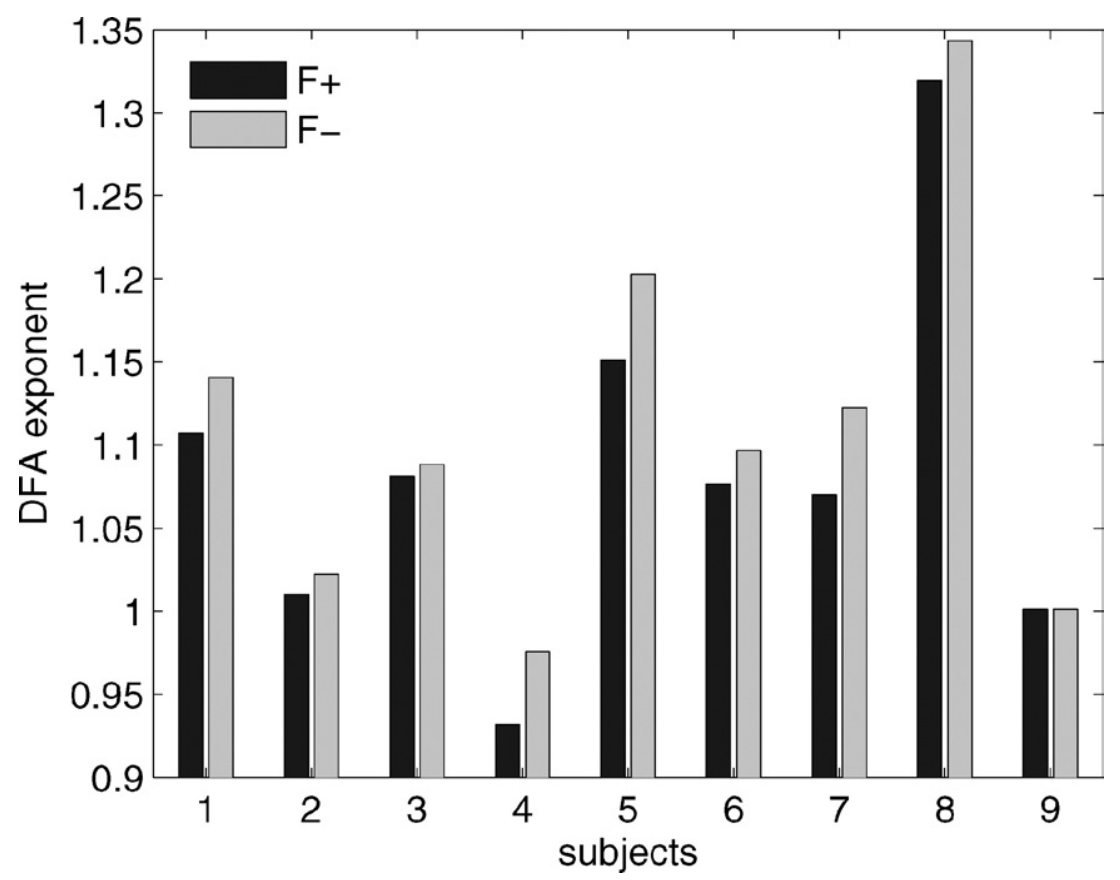

Fig. 7. Averages of single-subject DFA exponents over the electrodes belonging to the cluster showing significant feedback modulation. Black bars (gray bars) refer to positive (negative) feedback condition. The average exponent is higher for negative feedback than for positive feedback in eight subjects out of nine, and almost equal in the remaining subject.

area in which scaling exponents are significantly higher after negative feedback than after positive feedback
$(P<0.04)$. This cluster almost overlaps with the one emerging from the main analysis, confirming the previous
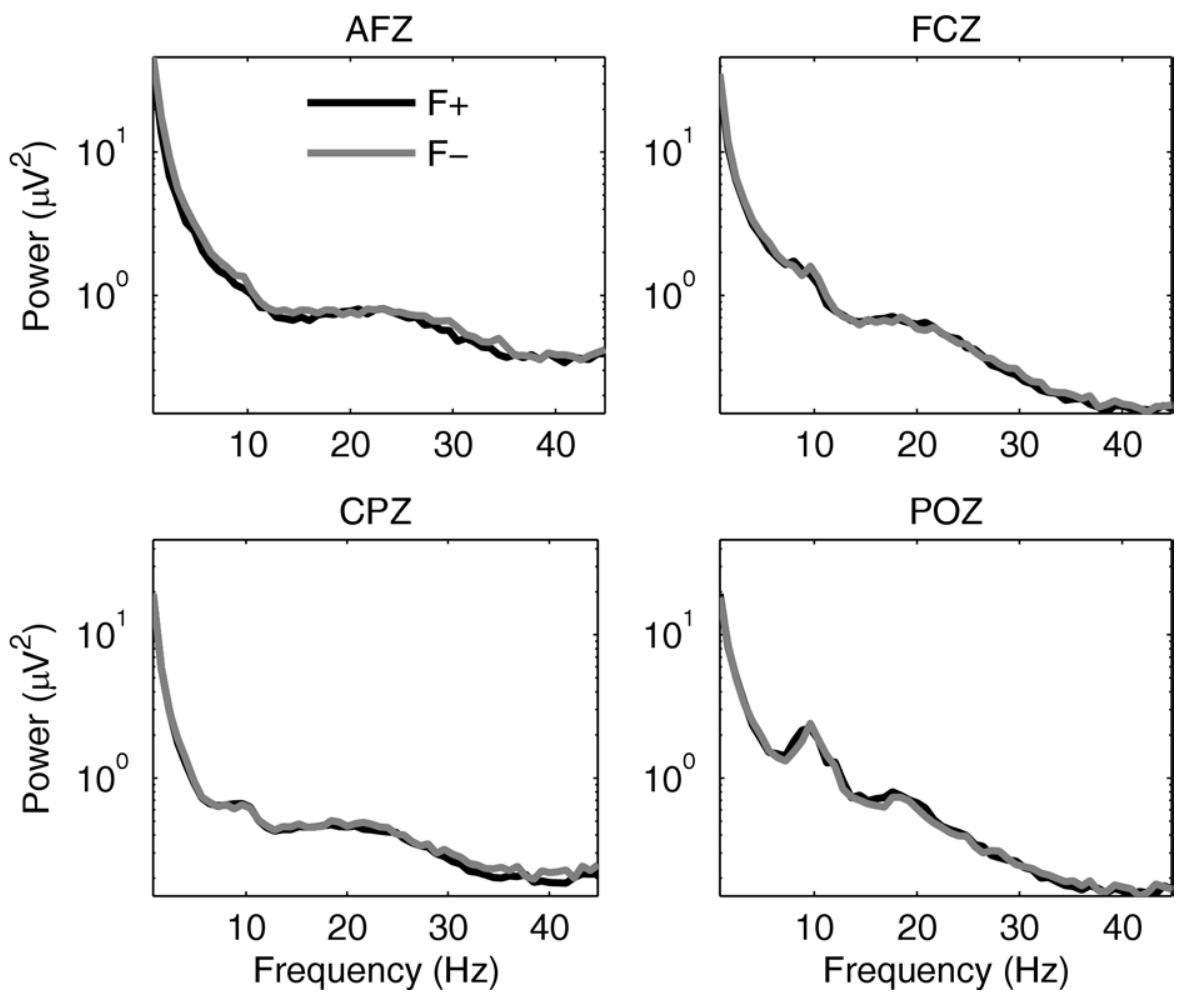

Fig. 8. Semilogarithmic plot of power spectrum as a function of frequency for positive (black line) and negative (gray line) feedback conditions relative to electrodes AFZ, FCZ, CPZ and POZ, subject 5. A power-law decrease at low frequencies followed by an alpha (10 Hz) peak in FCZ, CPZ and POZ is evident. No clear feedback modulation is visible. 

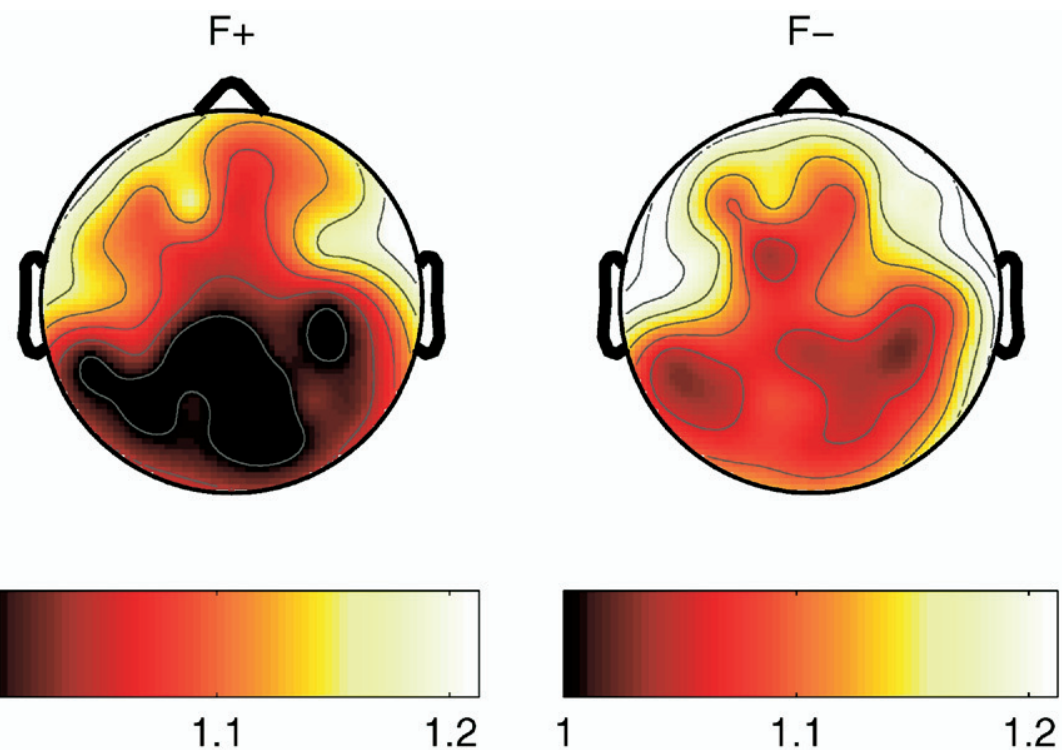

1.1

1.2
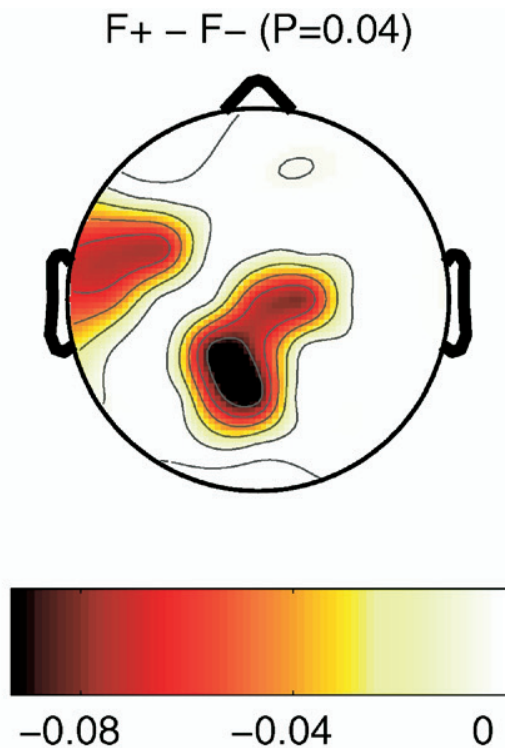

1

Fig. 9. Topography of the DFA scaling exponents averaged across all subjects for positive (left-hand scalp) and negative (middle scalp) feedback conditions. The right-hand scalp shows the topography of the difference of the averaged scaling exponents between the two conditions for the set of electrodes for which the scaling exponents are significantly different as evaluated by CRA $(P=0.04)$. Negative feedback scaling exponents are significantly higher than positive feedback ones in a wide brain area largely overlapping the one emerged from the main analysis. A zero difference is assigned to the electrodes not belonging to this set.

result. Residual EM surviving the EM correction algorithm may contribute to the high values of a few fronto-lateral scaling exponents emerging from the main analysis, and since they are also modulated by feedback, to the significant difference in the most frontal electrodes. However, the large overlap of the posterior and left anterior areas emerging from the two analyses strongly suggests that feedback modulation on these wide regions is largely independent from EM.

\section{DISCUSSION}

The main finding of this study is that the scale-free temporal dynamics of the brain electrical activity during a HT task is modulated by performance feedback: negative feedback elicits a higher degree of temporal scaling (i.e. a higher DFA scaling exponent) than positive feedback. We thus showed that specific task demands modulate the scale-free dynamics of the associated ongoing brain activity.

The two feedback conditions significantly differed at several electrodes clustered around two main areas: a left anterior and a middle posterior one. However, the topographic pattern of the scaling exponents was similar in the two conditions. This suggests that the brain activity modulated by feedback is widely distributed across different areas. This finding is consistent with the results of our previous study (Papo et al., 2006), in which intracranial sources of activity at various narrow-band frequencies were estimated in the 100-400 ms time-window following feedback onset. Positive and negative feedback were associated to early parahippocampo-cingular sources of alpha oscillations, and to late partially overlapping neural circuits, comprising regions in prefrontal, cingular, and temporal cortices, but operating at feedback-specific latencies and frequencies. However, given the non-linear nature of the DFA measure and the extended temporal range on which it is performed, inferences about the sources should remain highly speculative. Heuristically, higher exponent values for negative feedback can be explained in the following terms: following negative feedback, subjects ought to find a new hypothesis to test, after the previously tested one has been falsified. Contrary to the activity following positive feedback, subjects need to explore a potentially infinite space in order to select a new hypothesis to test. To explore this space in a more efficient manner, they need to keep track of their previous steps. Evidence from statistical physics suggests that efficient random searches under these conditions are characterized by scaling properties similar to those found in this paper (Viswanathan et al., 1999). The associated electrical brain activity may then reflect this aspect by modulating the way the system weights its own past as in the present study or by increasing the time-span during which the signal keeps track of its own past. Alternatively, the modulation of the scaling exponent could reflect less specific aspects of the task, like task difficulty or arousal. Further studies are needed to investigate which aspects of such a complex cognitive task are more relevant in modulating the scalefree behavior.

DFA scaling exponents, in the range 1-1.4, are compatible with the ones estimated in other studies using DFA on resting ongoing EEG signals (Watters, 1998; Lee et al., 2002), as well as using PSA (the two exponents being related by Eq. (9)) (Pereda et al., 1998; Freeman and Barrie, 2000; Le Van Quyen, 2003). Scaling exponents are homogeneous over the posterior half of the scalp, and 
increase toward the frontal areas (Fig. 6). This pattern is very similar in the two experimental conditions. The increase of frontal exponents is unlikely to be due to EM residual effects since it is also present in the eye-movement-free trials (Fig. 9). Previous studies did not investigate the topography of the scaling exponents. Since our study did not comprise a rest condition, we do not know whether stronger temporal correlations in frontal areas are specific to the HT task, or more generally related to ongoing brain activity.

Our results highlighted two different correlation regimes: while no scaling appears at short time scales, a clear power-law scaling emerges uniformly across electrodes and subjects for time windows larger than $150 \mathrm{~ms}$. A similar crossover around $100 \mathrm{~ms}$ has been shown and extensively discussed in a study of spontaneous ongoing brain activity (Hwa and Ferree, 2002). This suggests that different time ranges are characterized by separable statistical properties (Varela, 1999). A different dynamical origin can therefore be inferred. The disruption at short time scales of the long-range correlations present at larger scales could stem from the large peak around $10 \mathrm{~Hz}$ (Hwa and Ferree, 2002), which is clearly visible in our data in the power spectrum of several posterior electrodes (Fig. 8). An alternative explanation has been proposed by Robinson (2003), who suggested a relationship between crossover and dendritic constants. However, Hwa and Ferree (2002) found that short time scales also showed a scaling regime although with a scaling exponent that was significantly different from the long-range one. This may suggest that the crossover around $100 \mathrm{~ms}$ is a general property of the ongoing brain activity connected to the dominant alpha rhythm. On one hand, the lack of short-range scaling in our data may indicate that, on top of the temporal structure of the ongoing activity, there are task/stimulus-specific neural processes that disrupt scaling in the short range. On the other hand, the emergence of scale-free dynamics at longer ranges may suggest that the effect of transitory stimulations is a local perturbation that does not destroy the long-range temporal structure of the ongoing activity. This view is reinforced by the study of Linkenkaer-Hansen et al. (2004), who found that brief passive nerve stimulations induce a decrease of the scaling exponent of longrange temporal correlations in alpha and beta oscillations, but not a disruption of the scaling.

Despite the short time range, surrogate data analysis shows that DFA is able to estimate the correct scaling. In addition, the absence of feedback modulation in any specific frequency band suggests that modulation of temporal scaling is genuine, and not influenced by any process having a characteristic time scale.

It must be pointed out that, by comparison to previous studies, scaling was examined on a relatively narrow range of time scales, though the widest allowed by the experiment. This limitation is hard to bypass when investigating the scale-free dynamics of task-related brain activity, which is often inherently short. However, we believe that this limitation does not invalidate the claim of scale-free behavior in the range that we were able to study: a large number of analogous studies on natural systems have been performed on similarly narrow ranges but are widely accepted as valid, as the limitation is inherent to natural systems (Avnir et al., 1998).

\section{Scale-free dynamics in ongoing brain activity}

The emergence of scale-free behavior in the observed EEG activity may be explained by several hypotheses. Power-law, scale-free dynamics could simply arise as a result of the joint action of multiple processes, each with its own characteristic length. This explanation would be coherent with the heterogeneity of the time scales of the cognitive processes involved in the present study.

A more sophisticated theory suggests that the brain is in a state called critical in analogy with the behavior of equilibrium physical systems in the neighborhood of phase transitions. The critical state is characterized by fluctuations at all spatial and temporal scales, a phenomenon known as self-organized criticality (Bak et al., 1987, 1988; Maslov et al., 1994) which represents a good compromise between high susceptibility to perturbations and slowly decaying structural memory (Linkenkaer-Hansen et al., 2001; Bak et al., 1988; Linkenkaer-Hansen et al., 2004). Evidence for the differential modulation of temporal scaling of oscillations at several frequency bands by different cognitive tasks (Bhattacharya and Petsche, 2001; Popivanov et al., 2006) together with the results from the present study would indicate that external stimuli can modify the attitude of the system to respond to task-specific demands, and can modulate the way the signal keeps a memory of its own past, redefining the value of each moment to answer current task-specific needs (Hopfield and Brody, 2001). Our results further suggest that the modulation of the scale-free temporal dynamics of the ongoing activity can be driven by a discrete sensory stimulus within the same complex cognitive task.

The statistics underlying scale-free dynamics in EEG signals are poorly explored, probably due to their high non-stationarity. The link between scaling of DFA residuals and the statistical properties of the time series has been proved analytically only in the classical regime of weak chaos (Taqqu et al., 1995; Heneghan and McDarby, 2000), where the autocorrelation function decays as a power law. However, the behavior of DFA and PSA in the non-ergodic regime where the autocorrelation function is not defined anymore is not supported by a clear theoretic analysis yet. A recent study shows how non-ergodicity influences the averaging, causing other estimation methods to fail, and why the scaling exponents estimated by DFA are not affected by this effect (Kalashyan et al., in press).

\section{Neural correlates of reasoning}

The physiological basis of scale-free behavior of ongoing EEG oscillations has been suggested to be represented by neuronal activity-dependent plasticity operating at temporal scales within the scaling range, which would modify in a cumulative way the patterns of functional network connectivity (Azouz and Gray, 1999). In particular, synaptic plasticity has been proposed as a possible basis for the 
emergence of critical states of neural network's oscillatory activity (Linkenkaer-Hansen et al., 2001). The dynamical state of the synapse represents a transient memory buffer of the most recent segment of spike train previously sent to the synapse, and external stimuli may act by modulating this sliding window into the past (Maass et al., 2002). Feedback may modulate scaling behavior by acting on excitatory subthreshold activity, which dominates ongoing activity (Freeman and Barrie, 2000; Davidsen and Schuster, 2000; Tsuda, 2001), thereby varying the effective dimension of neuronal dynamics (Tsuda, 2001). Feedback could affect threshold levels, e.g. by modulating neuronal excitability, or the threshold dynamics of neuronal activity (Richardson et al., 2003), e.g. by modulating synaptic noise.

At different spatial and temporal scales, feedback could also exert its action on tonic neuromodulatory mechanisms. Following Papo et al. (2003), we suggest that positive feedback may modulate subsequent reward-related responses by regulating tonic dopamine release, while negative feedback may modulate tonic noradrenaline activity and the overall level of available neuronal energy, thereby having an impact on both memory and affective processes that can be activated by the system (Berridge and Waterhouse, 2003).

\section{CONCLUSION}

In conclusion, we have shown that the scale-free dynamics of the brain electrical activity are modulated by performance feedback during the preparatory period of a HT task. This result shows that specific cognitive demands can modulate the long-range temporal scaling properties of the ongoing brain activity. We believe that investigating the modulation of long-range scale-free brain temporal dynamics by cognitive processing is a potentially valuable approach to gain new insights on many complex cognitive processes.

A common view in the cognitive neuroscience of higher brain function holds that cognitive acts originate in the dynamics of loosely coupled modules (Van Orden et al., 2003). The dynamics inside a module dominate interactions with other components, and observed behavior can be partitioned among these devices. However, the scaling of the EEG signal indicates that the various components of the cognitive process at study are not trivially separable (Tsuda, 2001) and that observed behavior is an emergent property of coherent neuronal phenomena active in a wide range of intertwined, not trivially separable temporal scales (Van Orden et al., 2003). Variations in background noise arising from the coordination of time scales represent the signature of the underlying cognitive process, the neural correlates of which are represented by neurophysiological activity at all the scales characterized by similar correlations, from the neuronal constants' scale to that of observed behavior. This approach offers new possibilities to bridge the gap between the macroscopic level of behavior and the complexity of brain activity, with task-related neurophysiological activities unfolding at all temporal scales.
Acknowledgments-We are grateful to Manuela Piazza for insightful discussions and comments on the manuscript. D.P. was supported by a Post-doctoral Fellowship of the Fondation pour la Recherche Médicale, Paris (France). This article is dedicated to the memory of one of the coauthors, Pierre-Marie Baudonnière.

\section{REFERENCES}

Avnir D, Biham O, Lidar D, Malcai O (1998) Is the geometry of nature fractal? Science 279(5347):39-40.

Azouz R, Gray CM (1999) Cellular mechanisms contributing to response variability of cortical neurons in vivo. J Neurosci 19(6): 2209-2223.

Bak P, Tang C, Wiesenfeld K (1987) Self-organized criticality: An explanation of the 1/f noise. Phys Rev Lett 59(4):381-384.

Bak P, Tang C, Wiesenfeld K (1988) Self-organized criticality. Phys Rev A 38(1):364-374.

Berridge CW, Waterhouse BD (2003) The locus coeruleus-noradrenergic system: modulation of behavioral state and state-dependent cognitive processes. Brain Res 42(1):33-84.

Bhattacharya J, Petsche $H$ (2001) Universality in the brain while listening to music. Proc R Soc Lond B 268:2423-2433.

Buiatti M, Grigolini P, Palatella L (1999) A nonextensive approach to the entropy of symbolic sequences. Phys A 268(1-2):214-224.

Buldyrev SV, Goldberger AL, Havlin S, Mantegna RN, Matsa ME, Peng CK, Simons M, Stanley HE (1995) Long-range correlation properties of coding and noncoding DNA sequences: GenBank analysis. Phys Rev E 51(5):5084-5091.

Davidsen J, Schuster HG (2000) 1/f(alpha) Noise from self-organized critical models with uniform driving. Phys Rev E 62(5):6111-6115.

Elliott R, Frith CD, Dolan RJ (1997) Differential neural response to positive and negative feedback in planning and guessing tasks, Neuropsychologia 35(10):1395-1404.

Freeman WJ, Barrie JM (2000) Analysis of spatial patterns of phase in neocortical gamma EEGs in rabbit. J Neurophysiol 84(3):12661278.

Gong P, Nikolaev AR, van Leeuwen C (2003) Scale-invariant fluctuations of the dynamical synchronization in human brain electrical activity. Neurosci Lett 336(1):33-36.

Gratton G, Coles MG, Donchin E (1983) A new method for off-line removal of ocular artifact. Electroencephalogr Clin Neurophysiol 55(4):468-484.

Heneghan C, McDarby G (2000) Establishing the relation between detrended fluctuation analysis and power spectral density analysis for stochastic processes. Phys Rev E 62(5):6103-6110.

Herzel H, Schmitt AO, Ebeling W (1994) Finite-sample effects in sequence-analysis. Chaos Solitons Fractals 4(1):97-113.

Holroyd C, Coles MGH (2002) The neural basis of human error processing: reinforcement learning, dopamine, and the errorrelated negativity. Psychological Review, 109:679-709.

Hopfield JJ, Brody CD (2001) What is a moment? Transient synchrony as a collective mechanism for spatiotemporal integration. Proc Natl Acad Sci U S A 98(3):1282-1287.

Hwa RC, Ferree TC (2002) Scaling properties of fluctuations in the human electroencephalogram. Phys Rev E 66(2):021901.

Kalashyan A, Buiatti M, Grigolini P (2007) Ergodicity breakdown and scaling from single sequences. Chaos Solitons Fractals, in press.

Lee JM, Kim DJ, Kim IY, Park KS, Kim SI (2002) Detrended fluctuation analysis of EEG in sleep apnea using MIT/BIH polysomnography data. Comput Biol Med 32(1):37-47.

Le Van Quyen M (2003) Disentangling the dynamic core: a research program for a neurodynamics at the large scale. Biol Res 36:67-88.

Linkenkaer-Hansen K, Nikulin VV, Palva JM, IImoniemi RJ (2001) Long-range temporal correlations and scaling behavior in human brain oscillations. J Neurosci 21(4):1370-1377.

Linkenkaer-Hansen K, Nikulin VV, Palva JM, Kaila K, IImoniemi RJ (2004) Stimulus-induced change in long-range temporal correla- 
tions and scaling behaviour of sensorimotor oscillations. Eur J Neurosci 19(1):203-211.

Maass W, Natschlager T, Markram H (2002) Real-time computing without stable states: a new framework for neural computation based on perturbations. Neural Comput 14(11):2531-2560.

Margolin G, Barkai E (2005) Nonergodicity of blinking nanocrystals and other Lévy processes. Phys Rev Lett 94:080601.

Maslov S, Paczuski M, Bak P (1994) Avalanches and 1/f noise in evolution and growth models. Phys Rev Lett 73(16):2162-2165.

Miltner WHR, Braun CH, Coles M (1997) Event-related brain potentials following incorrect feedback in a time estimation task: Evidence for a "generic" neural system for error correction. J Cogn Neurosci 9:788-798.

Monchi O, Petrides M, Petre V, Worsley K, Dagher A (2001) Wisconsin Card Sorting revisited: distinct neural circuits participating in different stages of the task identified by event-related functional magnetic resonance imaging. J Neurosci 21(19):7733-7741.

Muller SV, Moller J, Rodriguez-Fornells A, Munte TF (2005) Brain potentials related to self-generated and external information used for performance monitoring. Clin Neurophysiol 116(1):63-74.

Nikulin VV, Brismar T (2005) Long-range temporal correlations in electroencephalographic oscillations: relation to topography, frequency band, age and gender. Neuroscience 130:549-558.

Novikov E, Novikov A, Shannahoff Khalsa D, Schwartz B, Wright J (1997) Scale-similar activity in the brain. Phys Rev E 56(3): R2387-R2389.

Papo D, Baudonniere PM, Hugueville L, Caverni JP (2003) Feedback in hypothesis testing: an ERP study. J Cogn Neurosci 15(4): 508-522.

Papo D, Douiri A, Bouchet F, Bourzeix JP, Caverni JP, Baudonniere PM (2006) Time-frequency intracranial source localization of feedback-related EEG activity in hypothesis testing. Cereb Cortex [Epub ahead of print] doi:10.1093/cercor/bhl042.

Peng CK, Buldyrev SV, Havlin S, Simons M, Stanley HE, Goldberger AL (1994) Mosaic organization of DNA nucleotides. Phys Rev E 49(2):1685-1689.

Peng CK, Havlin S, Stanley HE, Goldberger AL (1995) Quantification of scaling exponents and crossover phenomena in nonstationary heartbeat time series. Chaos 5(1):82-87.

Pereda E, Gamundi A, Rial R, Gonzalez J (1998) Non-linear behaviour of human EEG: fractal exponent versus correlation dimension in awake and sleep stages. Neurosci Lett 250(2):91-94.
Popivanov D, Stomonyakov V, Minchev Z, Jivkova S, Dojnov P, Jivkov S, Christova E, Kosev S (2006) Multifractality of decomposed EEG during imaginary and real visual-motor tracking. Biol Cybern 94:149-156

Rangarajan G, Ding M (2000) Integrated approach to the assessment of long range correlation in time series data. Phys Rev $E$ 61(5A):4991-5001.

Reddi BAJ, Carpenter RHS (2000) The influence of urgency on decision time. Nat Neurosci 3:827-830.

Richardson MJ, Brunel N, Hakim V (2003) From subthreshold to firing-rate resonance. J Neurophysiol 89(5):2538-2554.

Robinson PA (2003) Interpretation of scaling properties of electroencephalographic fluctuations via spectral analysis and underlying physiology. Phys Rev E 67(3):032902.

Ruchsow M, Grothe J, Spitzer M, Kiefer M (2002) Human anterior cingulate cortex is activated by negative feedback: evidence from event-related potentials in a guessing task. Neurosci Lett 325(3): 203-206.

Stam CJ, de Bruin EA (2004) Scale-free dynamics of global functional connectivity in the human brain. Hum Brain Mapp 22(2):97-109.

Stanley HE, Amaral LAN, Gopikrishnan P, Ivanov PC, Keitt TH, Plerou $V(2000)$ Scale invariance and universality: organizing principles in complex systems. Physica A 281(1-4):60-68.

Takashima A, Jensen O, Oostenveld R, Maris E, van de Coevering M, Fernandez G (2006) Successful declarative memory formation is associated with ongoing activity during encoding in a distributed neocortical network related to working memory: An MEG study. Neuroscience 139(1):291-297.

Taqqu SM, Teverovsky V, Willinger W (1995) Estimators for longrange dependence. Fractals 3(4):785-798.

Tsuda I (2001) Toward an interpretation of dynamic neural activity in terms of chaotic dynamical systems. Behav Brain Sci 24(5):793810; discussion 810-848.

Van Orden GC, Holden JG, Turvey MT (2003) Self-organization of cognitive performance. J Exp Psychol Gen 132(3):331-350.

Varela FJ (1999) The specious present. In: Naturalizing phenomenology (Petitot J, Varela FJ, Pachoud B, Roy JM, eds), pp 266-314. Stanford, CA: Stanford University Press.

Viswanathan GM, Buldyrev SV, Havlin S, da Luz MG, Raposo EP, Stanley HE (1999) Optimizing the success of random searches. Nature 401(6756):911-914.

Watters PA (1998) Fractal structure in the electroencephalogram. Complexity 5 . 